\title{
Research on Optimization of Freight Train ATO Based on Elite Competition Multi-Objective Particle Swarm Optimization
}

\author{
Lingzhi Yi' ${ }^{1}$, Renzhe Duan1', Wang Li², Yihao Wang1, Dake Zhang1, Bo Liu' ${ }^{1}$ \\ ${ }^{1}$ Hunan Province Engineering Research Center for Multi-Energy Collaborative Control Technology, College of Automation and \\ Electronics Information, Xiangtan University, Xiangtan, China \\ ${ }^{2}$ The State Key Laboratory of Heavy Duty AC Drive Electric Locomotive Systems Integration, Zhuzhou, China \\ Email: ylzwyh@xtu.edu.cn, 273405269@qq.com, hunanliwang@126.com,987922761@qq.com, 1912623288@qq.com, \\ 337620564@qq.com
}

How to cite this paper: Yi, L.Z., Duan, R.Z., Li, W., Wang, Y.H., Zhang, D.K. and Liu, B. (2021) Research on Optimization of Freight Train ATO Based on Elite Competition Multi-Objective Particle Swarm Optimization. Energy and Power Engineering, 13, 41-51.

https://doi.org/10.4236/epe.2020.134B005

Received: February 7, 2021

Accepted: April 5, 2021

Published: April 8, 2021

\begin{abstract}
In view of the complex problems that freight train ATO (automatic train operation) needs to comprehensively consider punctuality, energy saving and safety, a dynamics model of the freight train operation process is established based on the safety and the freight train dynamics model in the process of its operation. The algorithm of combining elite competition strategy with multi-objective particle swarm optimization technology is introduced, and the winning particles are obtained through the competition between two elite particles to guide the update of other particles, so as to balance the convergence and distribution of multi-objective particle swarm optimization. The performance comparison experimental results verify the superiority of the proposed algorithm. The simulation experiments of the actual line verify the feasibility of the model and the effectiveness of the proposed algorithm.
\end{abstract}

\section{Keywords}

Freight Train Automatic Train Operation, Dynamics Model, Competitive Multi-Objective Particle Swarm Optimization Algorithm (CMOPSO), Multi-Objective Optimization

\section{Introduction}

Railway transport is an indispensable mode of transportation. With the continuous increase of the scale of the railway transport network in our country, the freight operation is becoming more and more onerous, and the requirements for the comprehensive performance of train operation are getting higher and higher. 
Among them, safety, punctuality, energy saving and other performance are particularly worthy of attention. Therefore, the research on the multi-objective optimization of trains can not only ensure the safety and energy-saving operation of trains, but also be of great economic significance to reduce energy consumption [1].

For the optimization of train operation process, a lot of research has been done at home and abroad. An improved genetic algorithm is proposed in [2]. The constrained problem is transformed into an unconstrained problem by penalty function method. According to the characteristics of train operation, the dynamic model of train operation process is established in [3], and the linear weight method and multi-objective genetic algorithm are used to solve it. In [4], the non-inferior solution is obtained by multi-objective mixed integer elite genetic algorithm, and the expected results are obtained. In view of the problem that the train speed track is easy to fall into local convergence in [5], an improved multi-objective hybrid optimization method based on comprehensive learning strategy is proposed, which has good advantages. In [6], a multi-objective particle swarm optimization algorithm is proposed to solve the multi-objective optimization problem. Reference [7] based on the subway train, the multi-objective particle swarm optimization algorithm is used to obtain the non-inferior solution of energy consumption and time in the ATO system, taking into account the comfort of passengers. In order to balance the convergence and distribution of multi-objective particle swarm optimization, an adaptive multi-objective particle swarm optimization algorithm is proposed in [8], which improves the convergence of the algorithm. In [9], a multi-objective particle swarm optimization algorithm based on decomposition is proposed under the framework of genetic algorithm MOEA/D.

Based on the above analysis, combined with the characteristics of freight train operation, taking the freight train interval running time and energy consumption as the optimal control objectives, train operation safety factors and dynamic equations as constraints, the dynamic model of the freight train operation is established. In order to solve the problem that the distribution and convergence of the traditional multi-objective particle swarm optimization algorithm cannot be well balanced, the elite competition strategy is introduced to replace the update strategy of the traditional particle swarm. The effectiveness of the algorithm is verified by experimental simulation results.

\section{Dynamic Model of Freight Train}

\subsection{Analysis on the Operation Process of Freight Train}

The basic operation process of the freight train is to overcome the basic resistance and additional resistance and run on the established line under the action of electric locomotive traction and braking force. The freight train is affected by many factors in the actual operation, and the force situation is more complicated. Considering that the freight train runs along the track direction, only the 
longitudinal force of the freight train is considered, in order to simplify the analysis. It is divided into traction force $F_{t}(v)$, braking force $F_{b}(v)$ and basic resistance of operation $w_{0}(v)$ and additional resistance of the line $g(x)$.

The basic running resistance of the freight train depends on many factors, and the empirical equation is usually obtained through a large number of experiments. Taking HXD1 electric locomotive as an example, the empirical equation of basic resistance as follows:

$$
w_{0}(v)=a+b v+c v^{2} .
$$

In Equation (1), $v$ is the freight train speed, $a, b$ and $c$ are basic resistance coefficients.

The resistance encountered by freight train under additional conditions (through ramps, curves, tunnels) is called additional resistance. Different from the basic resistance, the unit additional resistance acting on locomotives and rolling stock is the same under the same line condition. The calculation equation of unit additional resistance is as follows:

$$
\left\{\begin{array}{l}
w_{i}(x)=1000 \sin \alpha=i \\
w_{r}(x)=\frac{600}{R} \\
w_{s}(x)=0.00013 L_{s}
\end{array} .\right.
$$

In order to facilitate the calculation, the unit plus ramp resistance is defined as the sum of three units of additional resistance:

$$
g(x)=w_{i}(x)+w_{r}(x)+w_{s}(x) .
$$

In Equation (2) and Equation (3), $w_{i}$ is the additional resistance of the unit ramp, $w_{r}$ is the additional resistance of the unit curve, $w_{s}$ is the additional resistance of the unit tunnel, $i$ is the slope of the ramp per thousand, $R$ is the radius of the curve, $L_{s}$ is the length of the tunnel.

In the process of freight train operation, there are four operating conditions: traction, idling, cruising (traction and braking force take turns to realize the uniform speed of the freight train) and braking, as shown in Figure 1. The resultant force acting on the freight train is different under different working conditions. The equation for calculating the resultant force of the freight train under different working conditions is shown in Equation (4).

$$
\begin{cases}c=f_{t}-w_{0}-g & \text { Traction } \\ c=-w_{0}-g & \text { coasting. } \\ c=-w_{0}-g-f_{b} & \text { braking }\end{cases}
$$

In Equation (4), $c$ represents the total force received during the train operation, $f_{t}$ represents the unit traction force of the train, $w_{0}$ represents the basic resistance of the train operation unit.

For this reason, the train movement process can be expressed by the dynamic equations shown in Equation (5).

$$
\frac{d v}{d t}=\frac{g}{1000(1+\gamma)} c=\frac{g}{1000(1+\gamma)}\left(u_{t} f_{t}-u_{b} f_{b}-w_{0}-g\right) \quad \frac{d x}{d t}=v
$$




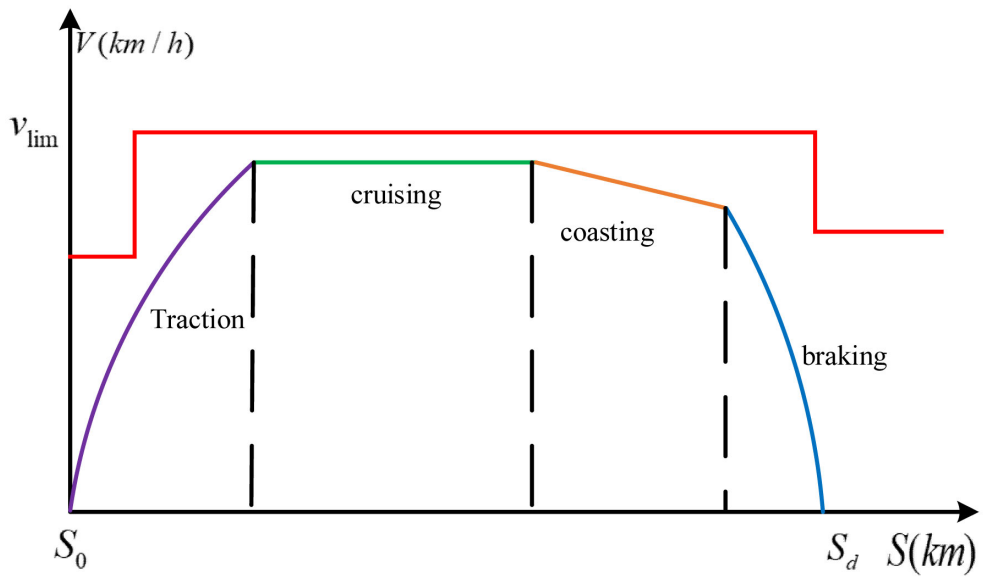

Figure 1. Four typical operating conditions of freight train ATO.

In Equation (5), $\gamma$ is the slewing mass coefficient, $x$ is the running distance of the train, $t$ is the running time of the train, $g$ is the acceleration of gravity, $c$ is the total force of the train, $u_{t} \in[0,1]$ and $u_{b} \in[0,1]$ respectively represent the traction force utilization coefficient and the braking force utilization coefficient.

\subsection{Multi-Objective Model Building}

Operation energy consumption includes the energy consumption of providing traction power for freight trains, the energy consumption needed to overcome resistance and the energy consumption of freight train auxiliary equipment, which accounts for more than $80 \%$ of the total energy consumption, so it is of great value to reduce the operation energy consumption. This paper only considers the energy consumed by the traction and braking process and auxiliary equipment of freight trains.

$$
E=\frac{\int F_{t} v d t}{\eta}+\lambda \int F_{b} v d t+A T
$$

In Equation (6), $\eta$ is the conversion coefficient of electric energy into mechanical energy during the freight train traction, $\lambda$ is the utilization rate of regenerative braking energy, $A$ is the auxiliary power, $T$ is the freight train operation time.

The running time is the time required for the freight train to run from the departure point to the end point, and the closer it is to the planned running time of the freight train, the higher the punctuality. The punctuality studied in this paper only refers to the running time between stations, without considering the stopping time. The evaluation model of punctuality of freight trains is as follows:

$$
\Delta T=T-T_{0} .
$$

In Equation (7), $T_{0}$ is the planned running time, $T$ is the actual running time, $\Delta T$ represents the deviation between the actual running time $T$ and planned running time $T_{0}$, and the smaller the deviation, the higher the punctuality. 
To sum up, the multi-objective optimization of freight train operation can be expressed as follows: under the conditions of satisfying the constraints of freight train operation safety, comfort and various kinematic models, the running time and the freight train energy consumption can be optimized as much as possible. The freight train dynamics model is as follows:

$$
f=\min \{E, \Delta T\} \quad \text { s.t. }\left\{\begin{array}{l}
v(0)=v(x)=0 \\
t(0)=0, t(x)=T \\
v(x) \leq v_{\max }(x) \\
a \leq a_{\max }
\end{array} .\right.
$$

In Equation (8), $x$ is the total distance of the freight train running, $v_{\max }(x)$ is the speed limit of the line at position $x, a$ is the freight train acceleration and $a_{\max }$ is the maximum allowable acceleration.

\section{Multi-Objective Particle Swarm Optimization Algorithm Based on Elite Competition Mechanism}

\subsection{Competitive Particle Swarm Optimization}

Cheng and Jin simulated the individual competition mechanism of survival of the fittest in biology and proposed competitive particle swarm optimization (PSO) in 2014. The algorithm assumes that the population size is (a multiple of 2) and initializes the population randomly within the search range; in each iteration, the population is randomly divided into groups by pairwise competition, and each group of particles are divided into winners and losers according to the size of the fitness value. The winners will directly enter the next generation, and the losers will learn and update their positions from the winners according to Equation (9).

$$
\left\{\begin{array}{l}
v_{l}(t+1)=r_{1}(t) v_{1}(t)+r_{2}(t)\left(x_{w}(t)-x_{l}(t)\right)+\varphi r_{3}(t)\left(\bar{x}(t)-x_{l}(t)\right) \\
x_{l}(t+1)=x_{l}(t)+v_{l}(t+1)
\end{array} .\right.
$$

where: $x_{1}(t)$, represents the location vector of the loser and the winner, respectively, $v_{1}(t)$ represents the speed vector of the loser, $t$ is the number of iterations, $r_{1}(t), r_{2}(t), r_{3}(t)$ are three random vectors uniformly distributed in the range of $\left[\begin{array}{ll}0 & 1\end{array}\right], \varphi$ is a control factor, control $\bar{x}(t)$ the impact on loser location updates.

\subsection{Multi-Objective Particle Swarm Optimization with Elite Competition Mechanism}

The multi-objective particle swarm optimization algorithm based on elite competition mechanism is mainly composed of two core parts: learning strategy based on competition mechanism and environment selection strategy.

\subsubsection{Learning Mechanism Based on Elite Competition}

The learning mechanism based on elite competition consists of elite particle selection strategy, paired competition strategy and particle learning strategy. The 
competitive strategy of the elite in pairs is described as follows:

The particles of $\mathrm{a}$ and $\mathrm{b}$ are two particles randomly selected from the elite solution set, the particles $\mathrm{p}$ are the current particles to be updated, $\theta_{1}$ and $\theta_{2}$ are the angles between particles $\mathrm{p}$ to be updated and elite particles a and $\mathrm{b}$. Calculate the angles $\theta_{1}$ and $\theta_{2}$, respectively, and compare $\theta_{1}$ and $\theta_{2}$. The particle with a small angle with particle $p_{i}$ is regarded as the winning particle, and the other as a competitive losing particle. As shown in Figure 2, where $\theta_{1}<\theta_{2}$, particle $\mathrm{a}$ is marked as the winning particle to guide the update of particle $\mathrm{p}$.

After the winning particle is selected, particle $p$ to be updated updates its position and speed attributes by learning from the winning particle. Assuming that the position attribute of particle $\mathrm{p}$ is $P_{i}$, the velocity attribute is $V_{i}$, and the position attribute of winning particle a is $P_{w}$, the velocity update formula and position update equation of particle $\mathrm{p}$ are as follows:

$$
V_{i}^{\prime}=R_{1} V_{i}+R_{2}\left(P_{w}-P_{i}\right) \quad P_{i}^{\prime}=P_{i}+V_{i}^{\prime} .
$$

In Equation (10), $R_{1}$ and $R_{2}$ is a randomly generated vector in the interval $\left[\begin{array}{ll}0 & 1\end{array}\right]$.

\subsubsection{Environment Selection Strategy}

The main purpose of the environmental selection strategy is to select the next generation population containing $\mathrm{N}$ particles from the parent and offspring, that is, population $\mathrm{P}$ and offspring $\mathrm{P}^{\prime}$. The truncation factor is mainly used to cut all the particles arranged in the front of the first Pareto until they are exactly $\mathrm{N}$ in size, and then replicate them to the next generation population.

\subsection{CMOPSO Algorithm to Solve the Model}

The elite competition mechanism MOPSO algorithm is used to solve the Dynamics model of freight train autopilot. As shown in Figure 3, the specific steps are as follows:

Step 1: Input basic simulation data and corresponding parameters.

Step 2: Initial population and takes the working conditions as the decision variable.

Step 3: The fitness value of particles is calculated by using the dynamic model in the process of freight train operation.

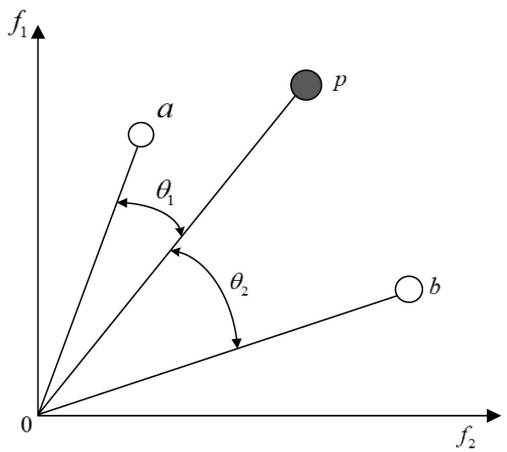

Figure 2. Competition mechanism between elite particles. 


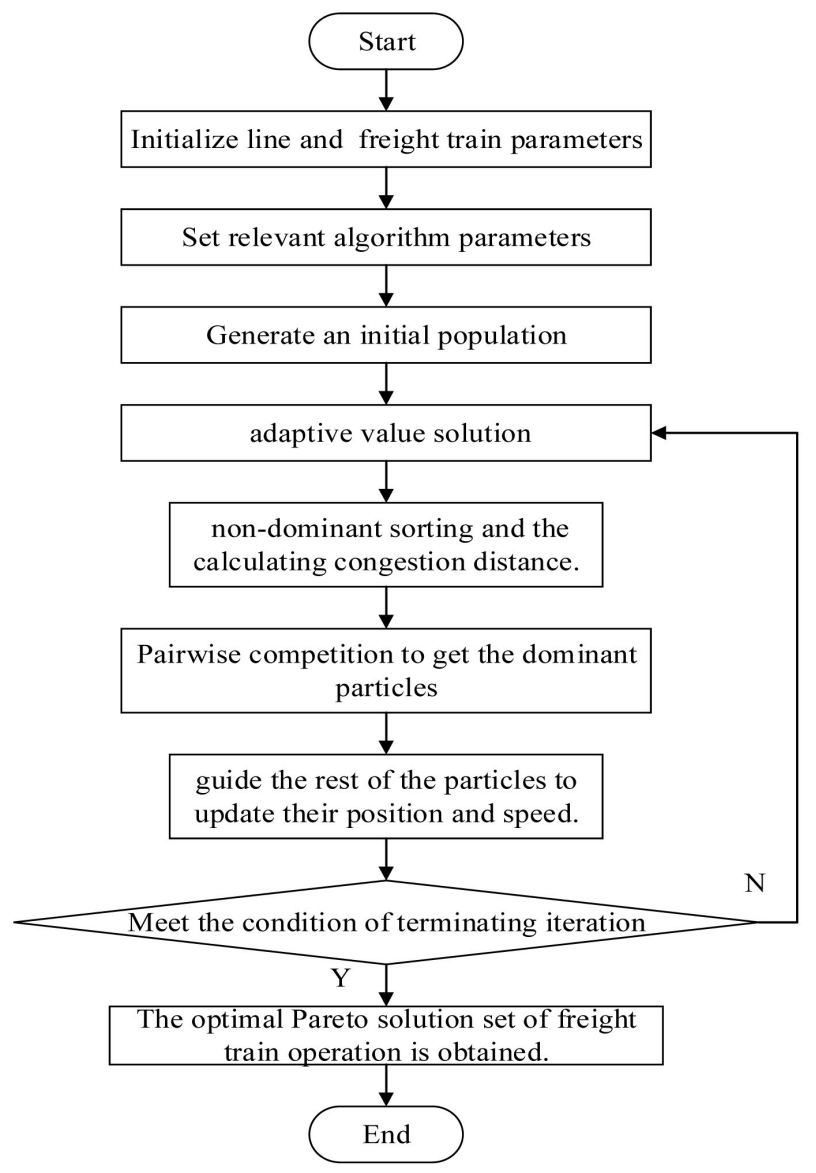

Figure 3. Flow chart of solving multi-objective optimization problem for freight train ATO.

Step 4: According to the elite competitive learning mechanism, the elite particles are selected from the population by the combination of non-dominant sorting and crowded distance to form the elite solution set $\mathrm{L}$, and then two particles are randomly selected to compete. The winning particles guide the update of the position and speed of the rest of the particles to be updated.

Step 5: According to the environmental selection strategy, the next generation population containing $\mathrm{N}$ particles is selected from the parent and offspring populations.

Step 6: Judge whether the stop condition is reached or not, move on to step 3 until the stop condition is met, and save the corresponding Pareto non-inferior solution.

\section{Simulation Analysis of Algorithm Example}

\subsection{Algorithm Performance Test}

In order to test the distribution and convergence of the Pareto frontier of the proposed algorithm, a comparative experiment is carried out with the MOPSO algorithm on the ZDT1 - ZDT3 problem. The experimental parameters are set as follows: the population size is 100 , the number of iterations is 300 , the size of the 
elite solution set is 10, and the algorithm is repeated for 30 times.

The Pareto front of CMOPSO algorithm on ZDT1 and ZDT2 is shown in Figure 4, from which we can see that the solution set obtained by CMOPSO optimization is more uniform than that of MOPSO, and each curve can better reflect the uniform distribution of solutions.

\subsection{Simulation Analysis of an Example}

Based on an actual line with a total length of $25 \mathrm{~km}$, a simulation study is carried out on HXD1 electric locomotive. The parameters of the freight train are shown in Table 1. Set the running time to $1250 \mathrm{~s}$ and the time margin to $40 \mathrm{~s}$. The initial population size of the algorithm is 50 , and the maximum number of iterations is 300. The simulation experiment is carried out under MATLAB.

MOPSO algorithm and CMOPSO algorithm are used to solve the freight train Dynamics model respectively, and the running results are shown in Table 2.

It can be seen from Table 2 that under the requirement of the freight train punctuality, the maximum and minimum energy consumption values obtained by CMOPSO are less than those obtained by MOPSO algorithm, indicating that the solution set obtained by CMOPSO algorithm is better, and the comprehensive optimization performance is better.
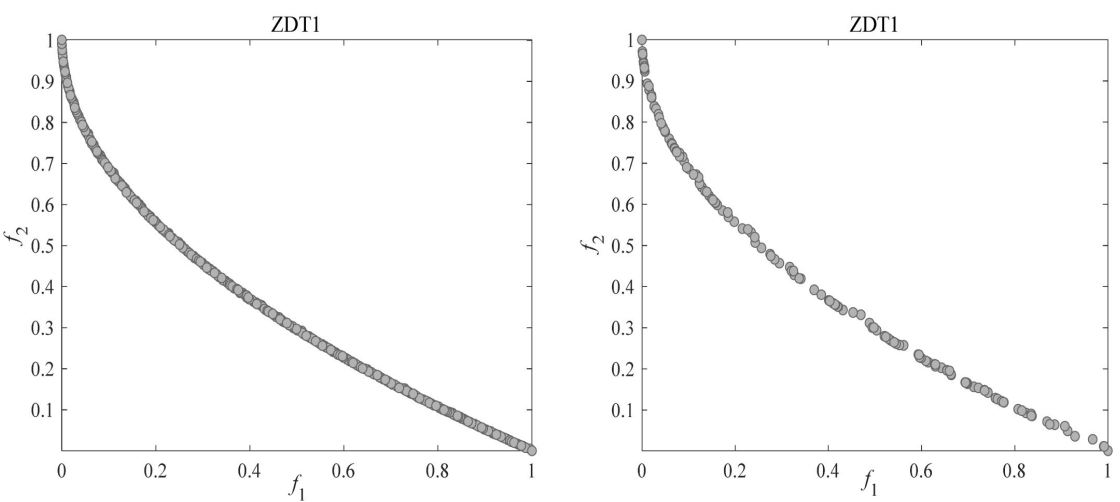

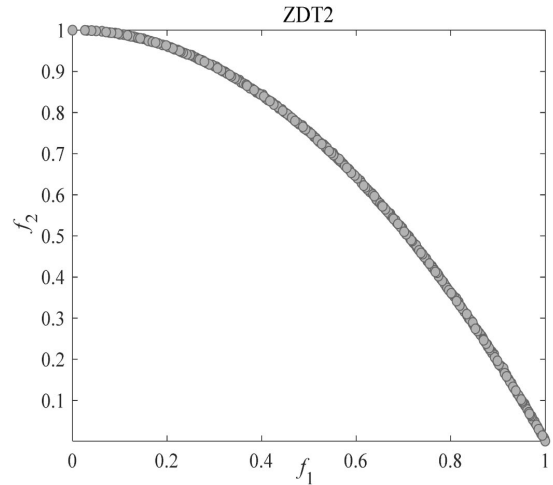

(a)

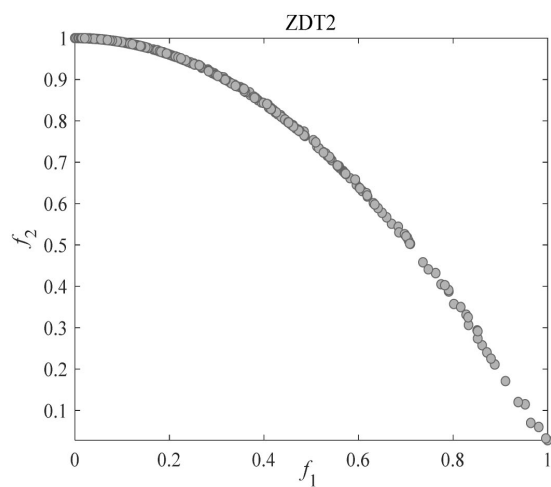

(b)

Figure 4. The non-dominant solution set obtained by the CMOPSO and MOPSO algorithms on the two-dimensional ZDTI and ZDT2 problem. (a) CMOPSO; (b) MOPSO. 
The curve with the best centralized punctuality solved by the two algorithms is shown in Figure 5. As can be seen from Figure 5, the freight train speed curves optimized by the two algorithms do not have the phenomenon of overspeed, and both reach the end point, so they meet the safety and punctuality constraints.

According to the calculation, the energy consumption and running time corresponding to the two speed distance curves are shown in Table 3. It can be seen that the time required by CMOPSO optimization is $1.7 \mathrm{~s}$ less than that optimized by MOPSO, and the operation energy consumption is reduced by $1.4 \%$.

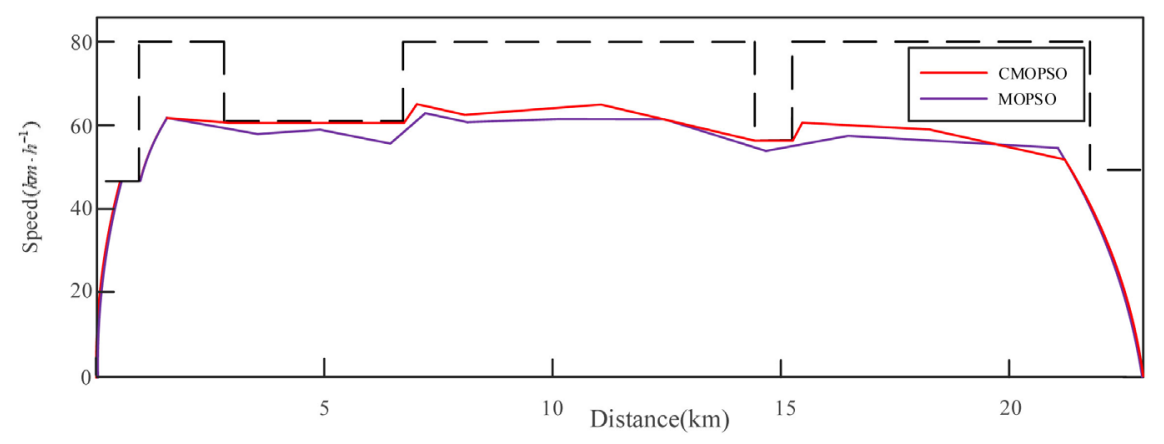

Figure 5. Speed-distance curve of freight train.

Table 1. The freight train parameters.

\begin{tabular}{cc} 
Parameters & Value \\
\hline The freight train mass $/ \mathrm{t}$ & 200 \\
Maximum speed $/\left(\mathrm{km} / \mathrm{h}^{-1}\right)$ & 120 \\
Maximum traction force $/ \mathrm{kN}$ & 760 \\
Maximum braking force $/ \mathrm{kN}$ & 461 \\
Energy consumption utilization $\eta$ & 0.9 \\
Regenerative braking feedback efficiency $\lambda$ & 0.85 \\
Basic unit resistance $/(\mathrm{N} / \mathrm{kN})$ & $1.2+0.0065 \mathrm{v}+0.000125 v^{2}$ \\
\hline
\end{tabular}

Table 2. Comparison table of simulation results of MOPSO and CMOPSO.

\begin{tabular}{ccc}
\hline Results & MOPSO & CMOPSO \\
Maximum running time/s & 1290 & 1293 \\
Minimum running time/s & 1231 & 1226 \\
Maximum energy consumption/kWh & 1021 & 793 \\
\hline Minimum energy consumption/ kWh & 805 & 1014 \\
\hline
\end{tabular}

Table 3. Operation results.

\begin{tabular}{ccc}
\hline Results & MOPSO & CMOPSO \\
\hline Energy consumption/kWh & 842.1 & 831.4 \\
Running time/s & 1251.3 & 1249.6 \\
\hline
\end{tabular}




\section{Conclusions}

In this paper, the elite competition mechanism MOPSO algorithm is introduced to replace the update strategy of the traditional multi-objective particle swarm optimization algorithm through the elite competition strategy.

The simulation analysis of the freight train operation example shows that the elite competition mechanism multi-objective particle swarm optimization algorithm has a certain optimization effect on freight train operation energy consumption, punctuality and other aspects, and can take into account the safety, punctuality and energy-saving operation of the freight train. The elite competitive MOPSO algorithm is used to optimize the freight train operation process, and a set of instructive Pareto optimal sets can be obtained, which provides a more real and feasible speed curve for the freight train operation.

\section{Acknowledgements}

This work was supported by the National Natural Science Foundation of China (61572416), Natural Science Foundation of Hunan Province (2020JJ6009), Open subject of The State Key Laboratory of Heavy Duty AC Drive Electric Locomotive Systems Integration.

\section{Conflicts of Interest}

The authors declare no conflicts of interest regarding the publication of this paper.

\section{References}

[1] Khmelnitsky, E. (2000) On an Optimal Control Problem of Train Operation. IEEE Transactions on Automatic Control, 45, 1257-1266. https://doi.org/10.1109/9.867018

[2] Liang, Y., Liu, H., Qian, C. and Wang, G. (2018) A Modified Genetic Algorithm for Multi-Objective Optimization on Running Curve of Automatic Train Operation System Using Penalty Function Method. International Journal of Intelligent Transportation Systems Research, 17, 74-87. https://doi.org/10.1007/s13177-018-0158-6

[3] Wang, L. Wang, X. and Sun, D. (2017) Multi-Objective Optimization Improved GA Algorithm and Fuzzy PID Control of ATO System for Train Operation. International Conference on Intelligent Computing for Sustainable Energy and Environment, Singapore, 23 August 2017, 13-22. https://doi.org/10.1007/978-981-10-6373-2_2

[4] Dullinger, C., Struckl, W. and Kozek, M. (2017) Simulation-Based Multi-Objective System Optimization of Train Traction Systems. Simulation Modelling Practice and Theory, 72, 104-117. https://doi.org/10.1016/j.simpat.2016.12.008

[5] Wang, L., Wang, X., Liu, K. and Sheng, Z. (2019) Multi-Objective Hybrid Optimization Algorithm Using a Comprehensive Learning Strategy for Automatic Train Operation. Energies, 12, 1882. https://doi.org/10.3390/en12101882

[6] Coello, C.A.C. and Lechuga, M.S. (2002) MOPSO: A Proposal for Multiple Objective Particle Swarm Optimization. Proceedings of the 2002 Congress on Evolutionary Computation, 1051-1056. https://doi.org/10.1109/CEC.2002.1004388 
[7] Dominguez, M., Fernandez-Cardador, A. and Cuclal, A.P. (2014) Multi Objective Particle Swarm Optimization Algorithm for the Design of Efficient ATO Speed Profiles in Metro Lines. Engineering Applications of Artificial, 29, 43-53. https://doi.org/10.1016/j.engappai.2013.12.015

[8] Hu, W. and Yen, G.G. (2015) Adaptive Multiobjective Particles Swarm Optimization Based on Parallel Cell Coordinate System. IEEE Transactions on Evolutionary Computation, 19, 1-18. https://doi.org/10.1109/TEVC.2013.2296151

[9] Peng, W. and Zhang, Q.A. (2008) A Decomposition-Based Multi-Objective Particle Swarm Optimization Algorithm for Continuous Optimization Problems. IEEE International Conference on Granular Computing, 534-537.

[10] Cheng, R. and Jin, Y. (2015) A Social Learning Particle Swarm Optimization Algorithm for Scalable Optimization. Information Sciences, 291, 43-60.

https://doi.org/10.1016/j.ins.2014.08.039 